Document downloaded from:

http://hdl.handle.net/10251/62423

This paper must be cited as:

García Cirujano, F.; Llabrés I Xamena, FX.; Corma Canós, A. (2012). MOFs as multifunctional catalysts: One-pot synthesis of menthol from citronellal over a bifunctional MIL-101 catalyst. Dalton Transactions. 41:4249-4254. doi:10.1039/c2dt12480g.

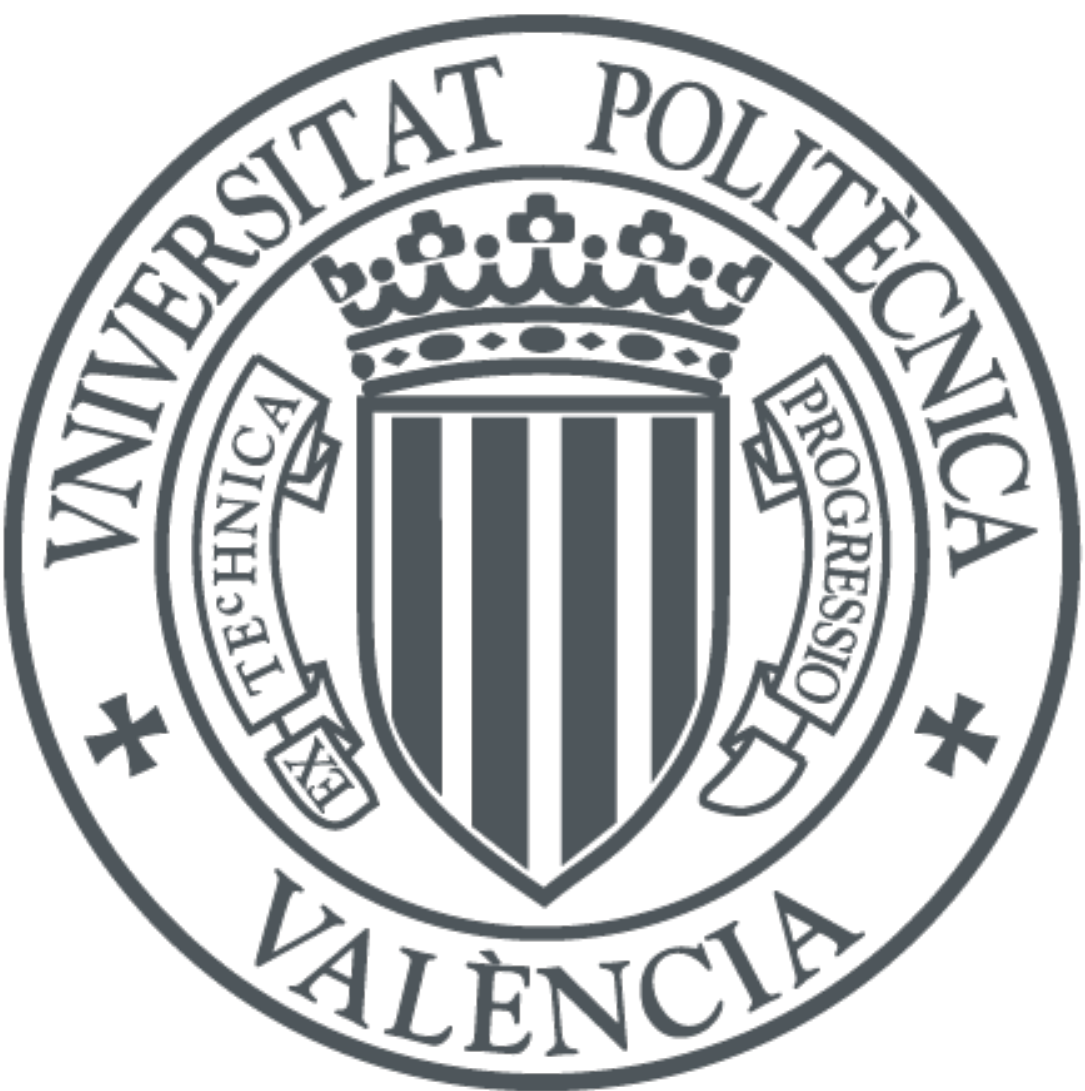

The final publication is available at

Copyright Royal Society of Chemistry

Additional Information 


\title{
MOFs as multifunctional catalysts: One-pot synthesis of menthol from citronellal over a bifunctional MIL-101 catalyst
}

\author{
F. G. Cirujano, F. X. Llabrés i Xamena* and A. Corma*
}

Instituto de Tecnología Química UPV-CSIC, Universidad Politécnica de Valencia, Consejo Superior de Investigaciones Científicas, Avda. de los Naranjos, s/n, 46022 Valencia, Spain

* Corresponding authors: e-mail: acorma@itq.upv.es

fllabres@itq.upv.es

fax: $\quad+34963877809$

\begin{abstract}
A bifunctional MOF catalyst containing coordinatively unsaturated $\mathrm{Cr}^{3+}$ sites and palladium nanoparticles (Pd@MIL-101) has been used for the cyclization of citronellal to isopulegol and for the one-pot tandem isomerization/hydrogenation of citronellal to menthol. The MOF was found to be stable under the reaction conditions used, and the results obtained indicate that the performance of this bifunctional solid catalyst is comparable with other state-of-the-art materials for the tandem reaction: Full citronellal conversion was attained over Pd@MIL-101 in $18 \mathrm{~h}$, with $86 \%$ selectivity to menthols and a diastereoselectivity of $81 \%$ to the desired (-)menthol, while up to $30 \mathrm{~h}$ were necessary for attaining similar values over Ir/H-beta under analogous reaction conditions.
\end{abstract}

\section{Introduction}

In recent years, Metal-Organic Framework compounds (MOFs) have attracted much interest for their potential as heterogeneous catalysts. ${ }^{1-3}$ Besides their high surface areas and pore volumes, which facilitate diffusion and the interfacial contact between the active site and the reagents, much of the potential of MOFs as heterogeneous catalysts relies on the possibility to fine-tune their chemical composition, pore dimensions and chemical environment inside the pores, by either selecting the appropriate building units or by post-synthesis modifications. ${ }^{4}$ The high flexibility in the design of MOFs facilitates the engineering of the catalytic sites of the material, 
since a number of different strategies can be used to introduce active centers. On one hand, the inorganic component can bear an active site, such as coordinatively unsaturated metal ions showing Lewis acidity or redox properties, or they can be used as anchoring points for introducing additional functionalities. ${ }^{5}$ On the other hand, the organic ligands can also hold functional groups, ${ }^{6,7}$ or they can be designed as coordination points to introduce metal active centers. ${ }^{8,9}$ Finally, the available pore space inside the MOFs can be efficiently used to introduce a variety of encapsulated species, including metal or metal oxide nanoparticles, ${ }^{10,11}$ polymers or discrete molecules, which can bring about new catalytic functions. However, in spite of this high tunability, the simultaneous introduction in MOFs of two or more active sites is an elusive subject, being the number of existing reports on multifunctional MOF catalysts very scarce to date. ${ }^{12-14}$ Vermoortele et al. ${ }^{12}$ have described the preparation of bifunctional acid-base MOFs, namely UiO-66 $\left(\mathrm{NH}_{2}\right)$, which contained basic amino groups close to coordinatively unsaturated $\mathrm{Zr}^{4+}$ acid sites generated in situ by controlled dehydroxylation. The authors used this material for the cross aldol-condensation of benzaldehyde and heptanal to form jasminaldehyde. For this reaction, the acid-base pairs work in a concerted way to activate simultaneously the two reactants, as was previously shown with other inorganic and organic catalysts with acid-base pairs. ${ }^{15-18}$. Similarly, Wu et al. ${ }^{13}$ prepared a lanthanide MOF containing Lewis acid $\mathrm{Tb}^{3+}$ and Lewis base triphenylamine sites, and used it for Knoevenagel an cyanosylalation reactions. Pan et al. ${ }^{14}$ reported on the use of Pd@MIL-101 as a bifunctional Lewis acid/hydrogenation catalyst, which allowed the one-pot synthesis of methyl isobutyl ketone from acetone and $\mathrm{H}_{2}$, through condensation, dehydration and hydrogenation. In the present work we have used a bifunctional hydrogenation/acid catalyst based on the $\mathrm{Cr}^{3+}$-containing MIL-101 $\mathrm{MOF}^{19}$ that shows excellent activity and selectivity for the one-pot two-steps synthesis of (-)-menthol from citronellal.

(-)-Menthol is a chemical compound widely used in flavouring and pharmaceutical applications. Its synthesis is usually carried out by multistep processes, such as the Haarmann and Reimer process starting from thymol, ${ }^{20}$ and the Takasago process starting from myrcene. ${ }^{21}$ However, another alternative method consists in the one-pot sequential transformation of citronellal (1) into isopulegol (2), ${ }^{22}$ followed by hydrogenation to menthol, as shown in Scheme 1. Besides isopulegol, citronellal cyclization can lead to three other stereoisomers $(\mathbf{3}, \mathbf{4}$ and $\mathbf{5})$, which after hydrogenation lead to four menthol stereoisomers, of which only that coming from isopulegol (2) is the desired product. Thus, it is important to determine the diastereoselectivity ratio to evaluate the performance of a catalyst for this reaction. To afford citronellal transformation into menthol in a one-pot, a bifunctional catalyst bearing acid and metal sites can be used. Jacobs et al. ${ }^{23,24}$ have reported that a 3\% Ir-impregnated H-BEA zeolite catalyzes the one-pot synthesis of menthol from citronellal in two steps. First, the cyclization step was carried out under $\mathrm{N}_{2}$ atmosphere, and then $\mathrm{H}_{2}$ was added to carry out the hydrogenation. Complete citronellal conversion was achieved after $30 \mathrm{~h}$, with $95 \%$ overall selectivity to the menthol 
isomers, of which $75 \%$ was the desired (-)-menthol. When this tandem reaction was performed in one-step (introducing $\mathrm{H}_{2}$ from the beginning), reaction times were shorter (16 h), but this resulted in lower selectivities, since citronellol and 3,7-dimethyl-octanol were also formed. ${ }^{23,24}$ Later reports described the use of Pt/H-beta zeolites ${ }^{25}$ and Pd-heteropolyacids ${ }^{26}$ for the same reaction of menthol synthesis from citronellal, or even starting from citral, which requires an additional hydrogenation step. ${ }^{27,28}$ These are excellent examples of catalysts based on supported metal nanoparticles in which the support not only affords a medium for dispersing the metal nanoparticles, but it does also participate actively in the catalytic process. In that sense, the high surface areas and pore volumes characteristic of some MOFs, make them excellent candidates for attaining high dispersion of metal nanoparticles. ${ }^{10}$ This, together with the possibility to introduce a second functionality, such as the presence of Lewis acid centers at the inorganic nodes, give MOFs the possibility to act as multifunctional catalysts.

\section{Scheme 1}

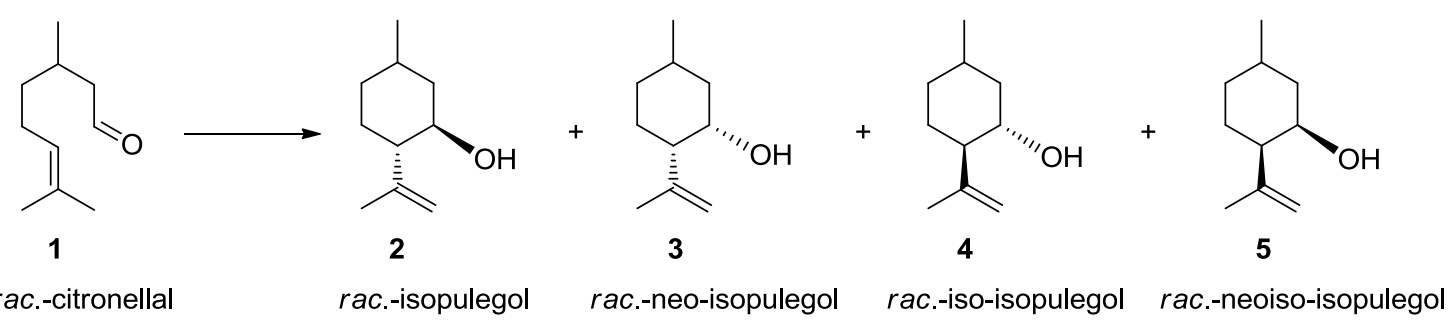

\section{Results and discussion}

We started our study by determining the performance of the $\mathrm{Cr}^{3+}$-MIL-101 support for the cyclization of citronellal. The reaction was carried out at $80^{\circ} \mathrm{C}$ under $\mathrm{N}_{2}$ atmosphere, using cyclohexane as solvent (see experimental), and the results are shown in Fig. 1a. As it can be seen there, full citronellal conversion was attained with $\mathrm{Cr}^{3+}$-MIL-101 after $18 \mathrm{hr}$. No products other than the isopulegols (2-5) were detected, while the selectivity for the isopulegol diastereomer (2) with respect to the other three diastereomers (3-5) was 74:26. These results demonstrate that the $\mathrm{Cr}^{3+}$ sites present in the MOF can catalyze this reaction. Indeed, it is known that MIL-101 features $\mathrm{Cr}^{3+}$ ions coordinated to a labile water molecule that can be easily removed to create a coordination vacancy on the metal ion. The resulting coordinatively unsaturated $\mathrm{Cr}^{3+}$ sites endow MIL-101 with Lewis acid character, in a similar way as in the case of $\left[\mathrm{Cu}_{3}(\mathrm{BTC})_{2}\right]\left(\mathrm{BTC}=1,3,5\right.$, benzenetricarboxylate) which has unsaturated $\mathrm{Cu}^{2+}$ ions and can catalize the above isomerization reaction. ${ }^{29}$ 

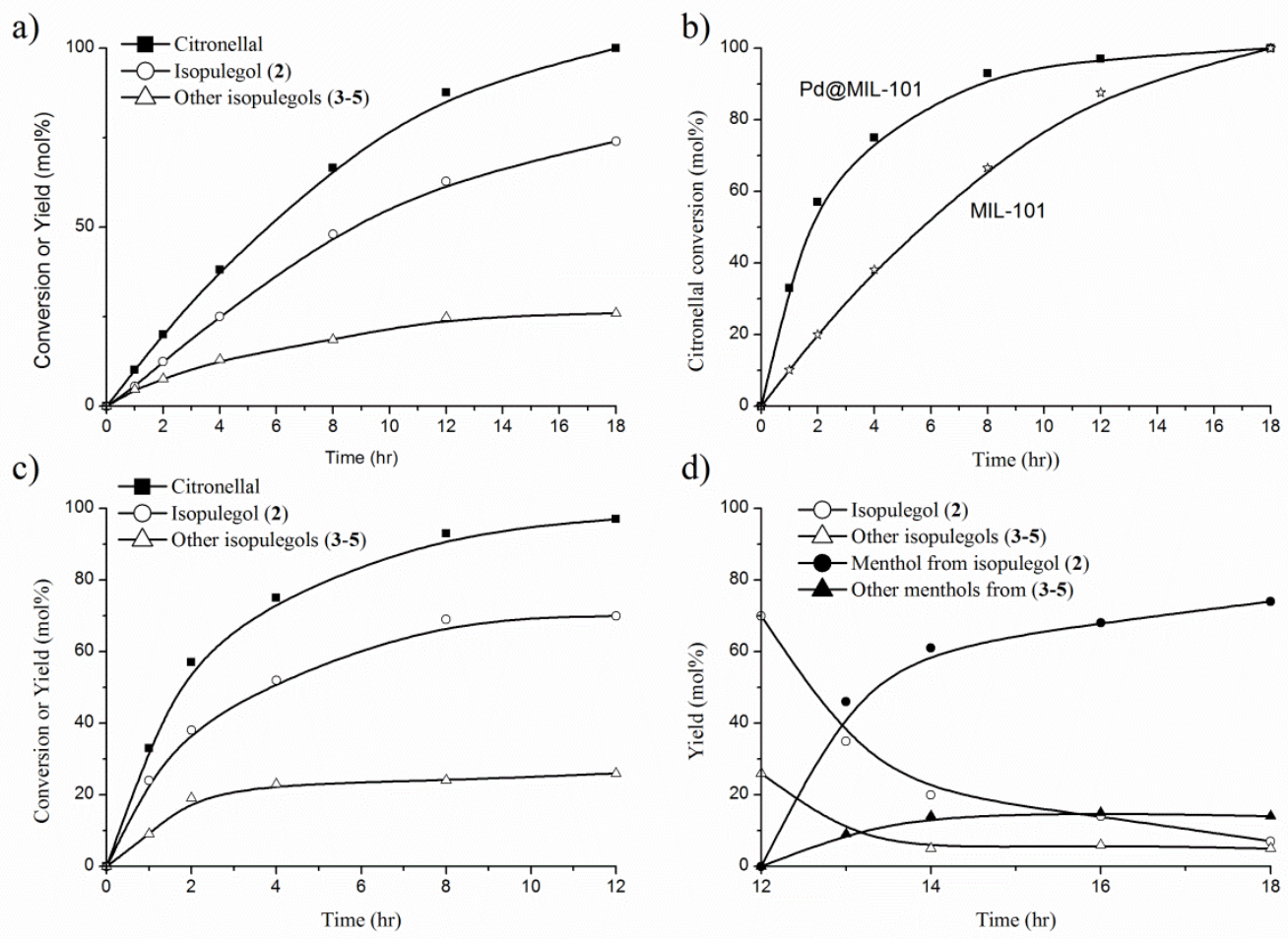

Figure 1. a) Time-conversion plots of citronellal (1) and yields to isopulegol (2) and other isopulegols (3-5) obtained using $\mathrm{Cr}^{3+}$-MIL-101 as catalyst. b) Comparison of the citronellal conversion obtained using $\mathrm{Cr}^{3+}$-MIL-101 or Pd@MIL-101 as catalyst. c) Time-conversion plots of citronellal (1) and yields to isopulegol (2) and other isopulegols (3-5) obtained using Pd@MIL-101 as catalyst. $d$ ) Time-evolution of products during hydrogenation of isopulegols to menthols.

As mentioned above, the most likely active sites in $\mathrm{Cr}^{3+}$-MIL-101 for the citronellal isomerization are coordinatively unsaturated $\mathrm{Cr}^{3+}$ sites, created upon removal of the apical $\mathrm{H}_{2} \mathrm{O}$ ligand. Nevertheless, some studies ${ }^{30-32}$ have revealed the presence of Bronsted type acidity in this class of solids, which could also contribute to the observed catalytic activity.

Since $\mathrm{Cr}^{3+}$-MIL-101 was active for the first step of the global process for converting citronellal into menthol, we could proceed to introduce the second catalytic function required. Then, the bifunctional Pd@MIL-101 material, containing coordinatively unsaturated $\mathrm{Cr}^{3+}$ Lewis acid sites and encapsulated Pd nanoparticles, was prepared (see experimental) and used as catalyst for the one-pot two steps transformation of citronellal into menthol. The first cyclization step was carried out under $\mathrm{N}_{2}$ atmosphere, at the same conditions as those used previously wity $\mathrm{Cr}^{3+}-\mathrm{MIL}-101$. The results obtained for the citronellal conversion and the yield to isopulegol (2) and other isopulegols (3-5) in the first reaction step using Pd@MIL-101 as catalyst are shown in Fig. 1c. As it can be observed in Fig. 1c, almost full conversion (97 mol\%) of citronellal can be achieved over Pd@MIL-101 after 12 hr, yielding isopulegols as the only 
detectable products, with a 73:27 diasteroselectivity ratio. One could expect that the presence of Pd nanoparticles could have a detrimental effect on the acid-catalyzed cyclization, taking into account the decrease of surface area and pore volume that occurs when Pd was incorporated inside the pores of MIL-101 (see Table S1 in Supporting Information). However, Pd@MIL-101 shows a slightly higher activity for the citronellal cyclization as compared with MIL-101 (see Fig. 1b). This difference can indicate the presence in Pd@MIL-101 of a fraction of $\mathrm{Pd}^{2+}$, not completely reduced during the hydrogenation of the palladium precursor, which can contribute to the acid-catalyzed citronellal cyclization. A similar observation was also described by Iosif et al. ${ }^{23}$ using iridium-impregnated $\mathrm{H}$-beta zeolite and an analogous interpretation was also given.

The second step of the tandem reaction to convert citronellal to menthol involves the hydrogenation of the isopulegol formed in the first step. This reaction is catalyzed by the encapsulated Pd nanoparticles. Thus, once the cyclization step was completed, the $\mathrm{N}_{2}$ atmosphere inside the reactor was replaced by $\mathrm{H}_{2}(0.8 \mathrm{MPa})$ while keeping the temperature unchanged. As can be observed in Figure 1d, hydrogenation of the cyclization products, isopulegol (2) and other pulegols (3-5), can be accomplished in about 6 hours, yielding selectively the corresponding menthols: $86 \%$ menthols ( $70 \mathrm{~mol} \%$ of the desired menthol coming from isopulegol (2) and $16 \mathrm{~mol} \%$ of other menthols), together with some small amounts of remaining non-hydrogenated isopulegols. This 70:16 ratio represents a diastereoselectivity of $81 \%$ for the (-)-menthol with respect to other menthols. From the results obtained it can be concluded that Pd@MIL-101 can be effectively used for the one-pot two-steps transformation of citronellal into menthol, attaining full citronellal conversion and an overall yield of the desired menthol of $74 \%$ after $18 \mathrm{hr}$.

The Pd@MIL-101 was found to be stable under the reaction conditions used, according to the XRD pattern and the ICP analysis of the solid recovered after the reaction (see Fig. S1 in Supporting Information). According to the TEM analysis, some aggregation of the Pd nanoparticles occur upon catalytic use, resulting in an average particle diameter of ca. $5 \mathrm{~nm}$ in the used catalyst (as compared to $3 \mathrm{~nm}$ in the fresh material), with a fraction of larger particles (see Fig. S2 in Supporting Information). Nevertheless the material still maintained a considerable fraction of specific surface area: $S_{\mathrm{BET}}=1620 \mathrm{~m}^{2} \mathrm{~g}^{-1}$. (see Table $\mathrm{S} 1$ in the Supporting Information). No leached Pd was detected in the solution by ICP analysis. Nevertheless, the contribution of homogeneous catalysis by eventually leached species after hot filtration was studied and the results in Fig. S3 in Supporting Information show negligible activity in the filtrate. In order to evaluate the recyclability of the catalyst, the solid recovered after the reaction was washed with cyclohexane and acetone and used in a second catalytic cycle. Similar results were obtained in this case, with only a small decrease of the activity during the cyclization step: $18 \mathrm{~h}$ were needed to attain full citronellal conversion, while only 12 $\mathrm{h}$ were necessary with the fresh catalyst. No appreciable decrease of activity was observed for 
the hydrogenation step. The selectivities to isopulegol in the first step and to menthol in the second step were practically the same with the fresh and with the used Pd@MIL-101.

Alternatively, the tandem transformation of citronellal into menthol can also be carried out in just one step: i.e., by performing the reaction under $\mathrm{H}_{2}$ atmosphere from the beginning. However, in this case the hydrogenation of the $\mathrm{C}=\mathrm{O}$ and $\mathrm{C}=\mathrm{C}$ bonds of citronellal can compete with the cyclization, leading to citronellol (6), 3,7-dimethyloctanal (7) and 3,7-dimethyloctanol (8), as shown in Scheme 2. The occurrence of these side reactions produces a decrease in the selectivity of citronellal to isopulegols and, hence, to the final desired menthols. When Pd@MIL-101 was used as catalyst (see experimental), we obtained full citronellal conversion after $20 \mathrm{~h}$. Unfortunately, the main product observed (60\% yield) under the reaction conditions used was 3,7-dimethyloctanol (8), which indicates that reduction of the $\mathrm{C}=\mathrm{O}$ and/or $\mathrm{C}=\mathrm{C}$ bonds is preferred over the cyclization. The combined selectivity to menthols amounted to $34 \%$, with a diastereoselectivity of $79 \%$ to the desired (-)-menthol (see Table 1B.2). Similar results were found for $\mathrm{Ir} / \mathrm{H}$-Beta when the reaction was performed at temperatures or $\mathrm{H}_{2}$ pressures lower than the optimum. ${ }^{23}$

\section{Scheme 2}
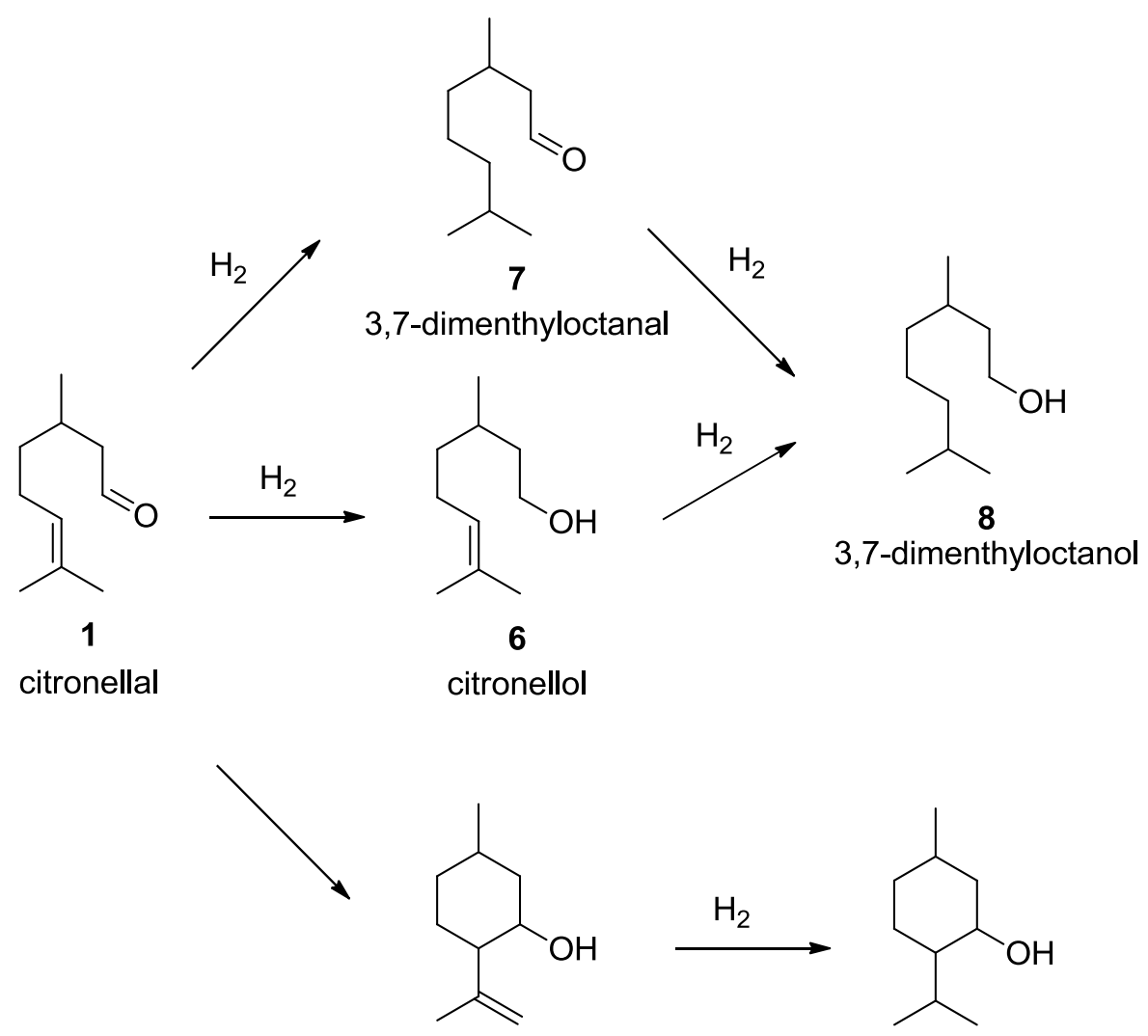

(2-5)

isopulegols

menthols 


\section{Comparison of Pd@MIL-101 and Cr3+-MIL-101 with other relevant catalysts}

At this point, and in order to put the activity and selectivity of the MOF catalyst into perspective, we have compared Pd@MIL-101 and the $\mathrm{Cr}^{3+}$-MIL-101 support with other related catalysts; i.e., Sn-Beta, Ir/H-Beta catalyst and the MOF $\left[\mathrm{Cu}_{3}(\mathrm{BTC})_{2}\right]$. A summary of the most relevant information is shown in Table 1 . We have compared the performance of the catalysts for both cyclization of citrolellal to isopulegols (Table 1A), and for the tandem cyclization/hydrogenation of citronellal to menthol (Table 1B), either in one-step or in twosteps. Note that the reaction conditions that we have chosen for this study (see experimental) are the same as those reported as the best for Ir/H-Beta: temperature, solvent, $\mathrm{H}_{2}$ pressure, and a citronellal to metal molar ratio of $\sim 425$. Therefore, comparison between Pd@MIL-101 and Ir/HBeta is straightforward.

Table 1. Summary of the catalytic results obtained for the isomerization of citronellal to isopulegol and tandem isomerization/hydrogenation of citronellal to menthol obtained with different catalysts.

A) Citronellal to isopulegol

\begin{tabular}{lrrlrccc}
\hline \multicolumn{1}{c}{ Catalyst } & $\begin{array}{r}\text { Temp. } \\
\left({ }^{\mathbf{o}} \mathbf{C}\right)\end{array}$ & $\boldsymbol{t}(\mathbf{h})$ & Solvent & $\begin{array}{c}\text { Conv. } \\
(\boldsymbol{\%})\end{array}$ & $\begin{array}{c}\text { Select. } \\
(\boldsymbol{\%})^{\boldsymbol{a}}\end{array}$ & $\begin{array}{c}\text { Diastereoselect. } \\
(\boldsymbol{\%})^{\boldsymbol{b}}\end{array}$ & Ref. \\
\hline $\mathrm{Cr}^{3+}$-MIL-101 & 80 & 18 & Cyclohexane & $>99$ & $>99$ & 74 & This work \\
Pd@MIL-101 & 80 & 12 & Cyclohexane & $>99$ & $>99$ & 73 & This work \\
Sn-Beta & 80 & 1 & Acetonitrile & 97 & $>99$ & 85 & 22 \\
H-Beta & 80 & 8 & Cyclohexane & $\sim 92$ & $>99$ & 75 & 24 \\
Ir/H-Beta & 80 & 8 & Cyclohexane & $\sim 96$ & $>99$ & 75 & 24 \\
{$\left[\mathrm{Cu}_{3}(\mathrm{BTC})_{2}\right]$} & 110 & $\sim 80$ & Toluene & $>99$ & $>99$ & $65-69$ & 29 \\
\hline
\end{tabular}

${ }^{a}$ Selectivity for the four diastereomeric isopulegols with respect to other products. ${ }^{b}$ Selectivity for the isopulegol diastereomer (2) with respect to the other three diastereomers (3-5).

B) One-pot citronellal to menthol

\begin{tabular}{lcccccc}
\hline B.1) Two-steps reaction: First step in $\mathrm{N}_{2}$ atmosphere, second step in $\mathrm{H}_{2}$ atmosphere & \\
\hline Catalyst & $\boldsymbol{p}\left(\mathbf{H}_{2}\right)(\mathbf{M P a})$ & $\boldsymbol{t}(\mathbf{h})$ & Conv. (\%) & Select. $(\boldsymbol{\%})^{\boldsymbol{a}}$ & $\begin{array}{c}\text { Diastereoselect. } \\
(\boldsymbol{\%})^{\boldsymbol{b}}\end{array}$ & Ref. \\
\hline Pd@MIL-101 & 0.8 & 18 & $>99$ & 86 & 81 & This work \\
\hline Ir/H-Beta & 0.8 & 30 & $>99$ & 95 & 75 & ${ }^{23}$ \\
\hline
\end{tabular}

B.2) Reaction in one-step: $\mathrm{H}_{2}$ supplied from the beginning

\begin{tabular}{lcccccc}
\hline \multicolumn{1}{c}{ Catalyst } & $\boldsymbol{p}\left(\mathbf{H}_{\mathbf{2}}\right)(\mathbf{M P a})$ & $\boldsymbol{t}(\mathbf{h})$ & Conv. (\%) & Select. $(\boldsymbol{\%})^{\boldsymbol{a}}$ & $\begin{array}{c}\text { Diastereoselect. } \\
(\boldsymbol{\%})^{\boldsymbol{b}}\end{array}$ & Ref. \\
\hline Pd@MIL-101 & 0.8 & 20 & $>99$ & 34 & 79 & This work \\
\hline Ir/H-Beta & 0.8 & 16 & $>99$ & 93 & 75 & 23 \\
\hline
\end{tabular}


As we have already commented above, Pd@MIL-101 is slightly more active for the citronellal isomerization to isopulegol that $\mathrm{Cr}^{3+}$-MIL-101 (see Fig. 1b), while both catalysts yield similar values of diastereoselectivities to isopulegol (2), of 73-74\%. From a simple inspection of Table 1A, it is evident that the activities and selectivities of both MIL-101 catalysts are much lower than those of Sn-Beta ${ }^{21}$, which allows full citronellal conversion in just $1 \mathrm{hr}$ with a significantly higher diastereoselectivity to isopulegol (2) of $85 \%$. The comparison of the MIL-101 catalysts and Sn-beta cannot be made directly, since the reactions are not done in exactly the same conditions, but they are rather similar: Same temperature and comparable amounts of catalyst, though different solvents were used in both cases. Nevertheless, the differences between Sn-Beta and the MIL-101 solids are so large in favor of the Sn-containing material that they cannot be attributed to the slightly different experimental conditions used, but to a clearly better performance of the tin catalyst. On the other hand, both $\mathrm{Cr}^{3+}$-MIL-101 and Pd@MIL-101 are better catalysts for citronellal cyclization than $\left[\mathrm{Cu}_{3}(\mathrm{BTC})_{2}\right]$, even when a significantly higher temperature was used for the reaction with copper catalyst. Less time was required to achieve full conversion over the MIL-101 catalysts, while yielding higher selectivities to the desired isopulegol product (2): e.g., 74\% selectivity for $\mathrm{Cr}^{3+}$-MIL-101 after $18 \mathrm{hr}$, as compared to $65-69 \%$ for $\left[\mathrm{Cu}_{3}(\mathrm{BTC})_{2}\right]$ after (at least) $48 \mathrm{hr}$, depending upon the preparation procedure. $^{29}$

The performance of Pd@MIL-101 and the support $\mathrm{Cr}^{3+}$-MIL-101 for the isomerization of citronellal is similar to that of Ir/H-Beta and the H-Beta support, both in terms of activity and selectivity to isopulegol (2), being the Beta catalysts only slightly more active than the MIL-101 MOFs (see Table 1A). In both systems, the metal-loaded catalysts are found to be slightly more active than the corresponding supports, which is attributed to the presence of a fraction of noncompletely reduced metal (Pd or Ir), which contributes to the acid-catalyzed cyclization of citronellal.

Concerning the tandem isomerization/hydrogenation reaction, the results obtained for Pd@MIL-101 were very different when the reaction was performed in two-steps or in one-step. When citronellal was allowed to isomerize under a $\mathrm{N}_{2}$ atmosphere until complete conversion before supplying $\mathrm{H}_{2}$, the tandem reaction proceeded smoothly to the formation of the desired menthol. In this case, the overall performance of the Pd@MIL-101 catalysts was comparable to the best results obtained with $\mathrm{Ir} / \mathrm{H}$-Beta. Considering that the reaction was performed with a citronellal to metal ratio of 425 with both Ir/H-beta and Pd@MIL-101, and that citronellal was quantitatively converted in both cases to isopulegols and menthols, one can calculate the mols of (-)-menthol produced per mol of noble metal and per catalytic cycle over the two materials, obtaining very similar values: 296 and 302 for Pd@MIL-101 and Ir/H-beta, respectively. However, longer reaction times were required for the beta catalyst $(30 \mathrm{~h})$ than for the MIL-101 solid (18 h). 
However, when a $\mathrm{H}_{2}$ atmosphere was used from the beginning of the reaction, the selectivity to menthols when Pd@MIL-101 was used as catalyst dropped significantly, being 3,7-dimethyloctanol (8) the main reaction product. This result indicates that hydrogenation of the $\mathrm{C}=\mathrm{C}$ and $\mathrm{C}=\mathrm{O}$ bonds is faster than the isomerization of citronellal to isopulegol. A similar result was also observed for the $\mathrm{Ir} / \mathrm{H}$-Beta when a temperature or a $\mathrm{H}_{2}$ pressure lower than the optimum was used. Overall, Ir/H-Beta was found to be a better catalyst (though still unsatisfactory) for the one-pot one-step conversion of citronellal to menthol than Pd@MIL-101, although the performances of the two catalysts were comparable when the reaction was performed in two-steps.

\section{Experimental}

\section{Catalysts preparation}

MIL-101 was prepared according to the reported procedures, ${ }^{19}$ while Pd@MIL-101 was obtained by impregnation of MIL-101 with $\mathrm{Pd}\left(\mathrm{NO}_{3}\right)_{2} \cdot 2 \mathrm{H}_{2} \mathrm{O}$ and reduction in a $\mathrm{H}_{2}$ flow. Details on the synthesis procedures can be found in the Supporting Information. Both materials were thoroughly characterized by XRD, $\mathrm{N}_{2}$ adsorption isotherms, TGA, TEM and ICP analysis. (see Supporting Information). Comparison of the XRD patterns of MIL-101 and Pd@MIL-101 were found to be practically identical, and both corresponded to the expected structure for the Crcontaining MOF (see Fig S1 in Supporting Information). According to the TEM analysis, Pd@MIL-101 was found to contain Pd nanoparticles of ca. $3 \mathrm{~nm}$, homogeneously distributed throughout the MIL-101 (see Fig. S2 in Supporting Information). According to the elemental analysis, the Pd content was $0.28 \mathrm{wt} \% \mathrm{Pd}$. These results are completely analogous to those reported for samples prepared by similar methods. ${ }^{14}$

\section{Catalytic reactions}

The citronellal isomerization reaction was performed at $80^{\circ} \mathrm{C}$ under a $\mathrm{N}_{2}$ atmosphere. $60 \mu \mathrm{l}$ of racemic citronellal and $30 \mathrm{mg}$ of MOF were placed inside a round bottom flask with $0.5 \mathrm{ml}$ of cyclohexane. The reaction was followed by GC-MS, using dodecane as external standard. Samples were analyzed by GC (Varian 3900), using a $30 \mathrm{~m}$ long and $0.25 \mathrm{~mm}$ i.d. capillary column HP-5 (5\% phenylmethylpolysiloxane). Retention times were compared with those of commercial standards.

For the tandem isomerization/hydrogenation reaction, the $\mathrm{N}_{2}$ atmosphere was replaced by a $\mathrm{H}_{2}$ atmosphere $\left(p\left(\mathrm{H}_{2}\right)=0.8 \mathrm{MPa}\right)$, upon completion of the isomerization reaction. Both $\mathrm{Cr}^{3+}$-MIL101 and Pd@MIL-101 were used in the reaction as-prepared, without any further pre-activation.

\section{Acknowledgements}

Financial support by Ministerio de Educación y Ciencia e Innovación (Project MIYCIN, CSD2009-00050; PROGRAMA CONSOLIDER. INGENIO 2009), Generalidad Valenciana 
(GV PROMETEO/2008/130) and the CSIC (Proyectos Intramurales Especiales 201080I020) is gratefully acknowledged.

\section{References}

1. A. Corma, H. Garcia and F. X. Llabrés i Xamena, Chem. Rev., 2010, 110, 4606-4655.

2. D. Farrusseng, S. Aguado and C. Pinel, Angew. Chem., Int. Ed., 2009, 48, 7502-7513.

3. J. Y. Lee, O. K. Farha, J. Roberts, K. A. Scheidt, S. T. Nguyen and J. T. Hupp, Chem. Soc. Rev., 2009, 38, 1450-1459.

4. Z. Q. Wang and S. M. Cohen, Chem. Soc. Rev., 2009, 38, 1315-1329.

5. M. Banerjee, S. Das, M. Yoon, H. J. Choi, M. H. Hyun, S. M. Park, G. Geo and K. Kim, J. Am. Chem. Soc., 2009, 131, 7524-7525.

6. J. Gascon, U. Aktay, M. D. Hernandez-Alonso, G. P. M. van Klink and F. Kapteijn, J. Catal., 2009, 261, 75-87.

7. S. Hasegawa, S. Horike, R. Matsuda, S. Furukawa, K. Mochizuki, Y. Kinoshita and S. Kitagawa, J. Am. Chem. Soc., 2007, 129, 2607-2614.

8. S. H. Cho, B. Q. Ma, S. T. Nguyen, J. T. Hupp and T. E. Albrecht-Schmitt, Chem. Commun., 2006, 2563-2565.

9. X. Zhang, F. X. Llabrés i Xamena and A. Corma, J. Catal., 2009, 265, 155-160.

10. M. Meilikhov, K. Yusenko, D. Esken, S. Turner, G. Van Tendeloo and R. A. Fischer, Eur. J. Inorg. Chem., 2010, 3701-3714.

11. A. Henschel, K. Gedrich, R. Kraehnert and S. Kaskel, Chem. Commun., 2008, 4192-4194.

12. F. Vermoortele, R. Ameloot, A. Vimont, C. Serre and D. E. De Vos, Chem. Commun., 2011, 47, 1521-1523.

13. P. Wu, J. Wang, Y. Li, C. He, Z. Xie and C. Duan, Adv. Funct. Mater., 2011, 21, 27882794.

14. Y. Pan, B. Yuan, Y. Li and D. He, Chem. Commun., 2010, 46, 2280-2282.

15. M. J. Climent, A. Corma, R. Guil-Lopez, S. Iborra and J. Primo, J. Catal., 1998, 175, 7079.

16. M. J. Climent, A. Corma, S. Iborra and A. Velty, J. Mol. Catal. A-Chem., 2002, 182-183, 327-342.

17. M. Boronat, M. J. Climent, A. Corma, S. Iborra, R. Montón and M. J. Sabater, Chem. Eur. J, 2010, 16, 1221-1231.

18. A. Corma, U. Diaz, T. Garcia, G. Sastre and A. Velty, J. Am. Chem. Soc., 2010, 132, 15011-15021.

19. G. Ferey, C. Mellot-Draznieks, C. Serre, F. Millange, J. Dutour, S. Surble and I. Margiolaki, Science, 2005, 309, 2040-2042.

20. J. Fleischer, K. Bauer and R. Hoop, DE 2109456, 1971.

21. S. Otsuka, K. Tani, T. Yamagata, S. Akutagawa, H. Kumobayashi and M. Yagi, EP Patent 68506, 1972.

22. A. Corma and M. Renz, Chem. Commun., 2004, 550-551.

23. F. losif, S. Coman, V. Parvulescu, P. Grange, S. Delsarte, D. E. De Vos and P. A. Jacobs, Chem. Commun., 2004, 1292-1293.

24. F. Neatu, S. Coman, V. Parvulescu, G. Poncelet, D. E. De Vos and P. A. Jacobs, Top. Catal., 2009, 52, 1292-1300.

25. P. Mertens, F. Verpoort, A.-N. Parvulescu and D. E. De Vos, J. Catal., 2007, 243, 7-13.

26. K. A. da Silva Rocha, P. A. Robles-Dutenhefner, E. M. B. Sousa, E. F. Kozhevnikova, I. V. Kozheznikov and E. V. Gusevskaya, Appl. Catal. A: Gen., 2207, 317, 171-174.

27. A. F. Trasarti, A. J. Marchi and C. R. Apesteguía, J. Catal., 2004, 224, 484-488.

28. A. F. Trasarti, A. J. Marchi and C. R. Apesteguía, J. Catal., 2007, 247, 155-165. 
29. L. Alaerts, E. Seguin, H. Poelman, F. Thibault-Starzyk, P. A. Jacobs and D. E. De Vos, Chem. Eur. J, 2006, 12, 7353-7363.

30. P. Horcajada, S. Surble, C. Serre, D. Y. Hong, Y. K. Seo, J. S. Chang, J. M. Greneche, I. Margiolaki and G. Ferey, Chem. Commun., 2007, 2820-2822.

31. U. Ravon, G. Chaplais, C. Chizallet, B. Seyyedi, F. Bonino, S. Bordiga, N. Bats and D. Farrusseng, ChemCatChem, 2010, 2, 1235-1238.

32. A. Vimont, H. Leclerc, F. Mauge, M. Daturi, J. C. Lavalley, S. Surble, C. Serre and G. Ferey, J. Phys. Chem. C, 2007, 111, 383-388. 


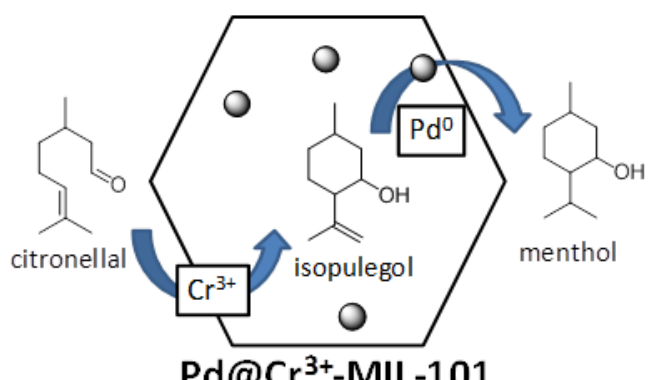

Pd@Cr ${ }^{3+}-\mathrm{MIL}-101$

A bifunctional MOF catalyst is used for the tandem one-pot synthesis of menthol from citronellal, with activities and diastereoselectivity comparable to state-of-the-art catalysts 


\section{SUPPORTING INFORMATION}

\section{A) Catalyst preparation}

Preparation of MIL-101: $\mathrm{Cr}^{3+}$-MIL-101 was prepared according to the reported procedure. ${ }^{\mathrm{i}}$ Briefly, $500 \mathrm{mg}$ of terephtalic acid and $800 \mathrm{mg} \mathrm{Cr}\left(\mathrm{NO}_{3}\right)_{3} \cdot 9 \mathrm{H}_{2} \mathrm{O}$ were dissolved in $15 \mathrm{~mL} \mathrm{H}_{2} \mathrm{O}$ and $40 \mu \mathrm{L} \mathrm{HF}(40 \%)$. The solution was placed inside an autoclave and kept at $200^{\circ} \mathrm{C}$ for $8 \mathrm{~h}$ in an oven. The resulting material was recovered by filtration, washed with DMF and hot ethanol, and dry at RT in air.

Preparation of Pd@MIL-101: The preparation procedure for this material was adapted from that reported by Pan et al. ${ }^{\mathrm{ii}}$ Briefly, $500 \mathrm{mg}$ of $\mathrm{Cr}^{3+}-\mathrm{MIL}-101$ were dried under a vacuum at $120^{\circ} \mathrm{C}$, and impregnated with $0.5 \mathrm{~mL}$ of an aqueous solution containing $10 \mathrm{mg} \mathrm{mL}^{-1}$ of $\operatorname{Pd}\left(\mathrm{NO}_{3}\right)_{2} \cdot 2 \mathrm{H}_{2} \mathrm{O}$. The solid was then dried in air and heat treated up to $200^{\circ} \mathrm{C}$ under a $\mathrm{N}_{2}$ atmosphere and kept at this temperature under a $\mathrm{H}_{2}$ atmosphere for additional $2 \mathrm{~h}$ to reduce the palladium precursor. According to ICP, the Pd content of this samples was $0.28 \mathrm{wt} \% \mathrm{Pd}$.

\section{B) Catalytic tests}

The citronellal isomerization reaction was performed at $80^{\circ} \mathrm{C}$ under a $\mathrm{N}_{2}$ atmosphere. $60 \mu \mathrm{l}$ of racemic citronellal and $30 \mathrm{mg}$ of MOF was placed inside a round bottom flask with $0.5 \mathrm{ml}$ of cyclohexane. The reaction was followed by GC-MS, using dodecane as external standard.

For the tandem isomerization/hydrogenation reaction, the $\mathrm{N}_{2}$ atmosphere was replaced by a $\mathrm{H}_{2}$ atmosphere $\left(p\left(\mathrm{H}_{2}\right)=0.8 \mathrm{MPa}\right)$. upon completion of the isomerization reaction.

\section{C) Textural properties of the catalysts}

Table S1

\begin{tabular}{|l|c|c|}
\hline Sample & $\mathbf{S}_{\text {BET }}\left(\mathbf{m}^{2} \mathbf{g}^{-\mathbf{1}}\right)$ & $\mathbf{V}_{\mathbf{p}}\left(\mathbf{c m}^{\mathbf{3}} \mathbf{g}^{\mathbf{- 1}}\right)$ \\
\hline $\mathrm{Cr}^{3+}$-MIL-101 & 2336 & 1.40 \\
\hline Pd@MIL-101 & 2182 & 0.97 \\
\hline Pd@MIL-101 used $^{a}$ & 1619 & 0.72 \\
\hline
\end{tabular}

${ }^{a}$ Material recovered after one catalytic cycle (tandem one-pot two steps isomerization/hydrogenation of citronellal to menthol at $80^{\circ} \mathrm{C}$ and $\left.p\left(\mathrm{H}_{2}\right)=0.8 \mathrm{MPa}\right)$. 


\section{D) XRD analysis}

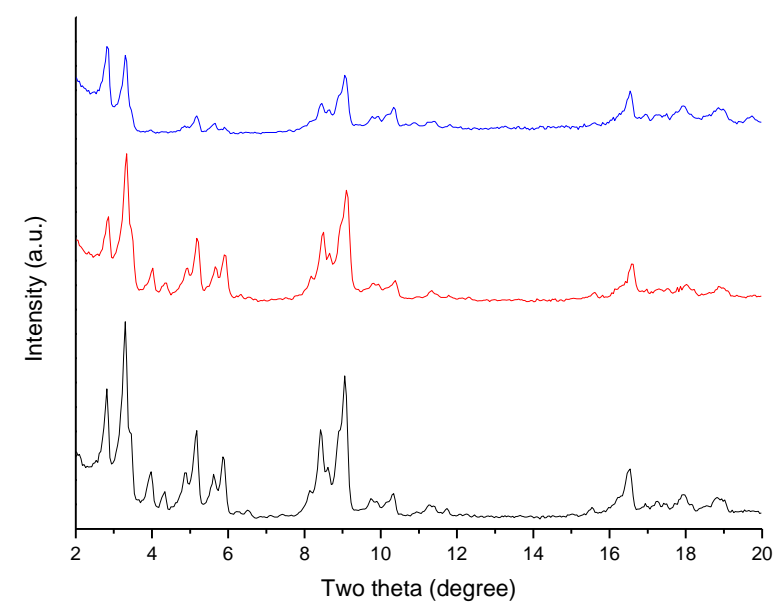

Fig. S1. XRD patterns (Cu K $\alpha$ radiation) of MIL-101 (black), Pd/MIL-101 before the reaction (red) and Pd/MIL-101 after the reaction (blue).

\section{E) TEM analysis}
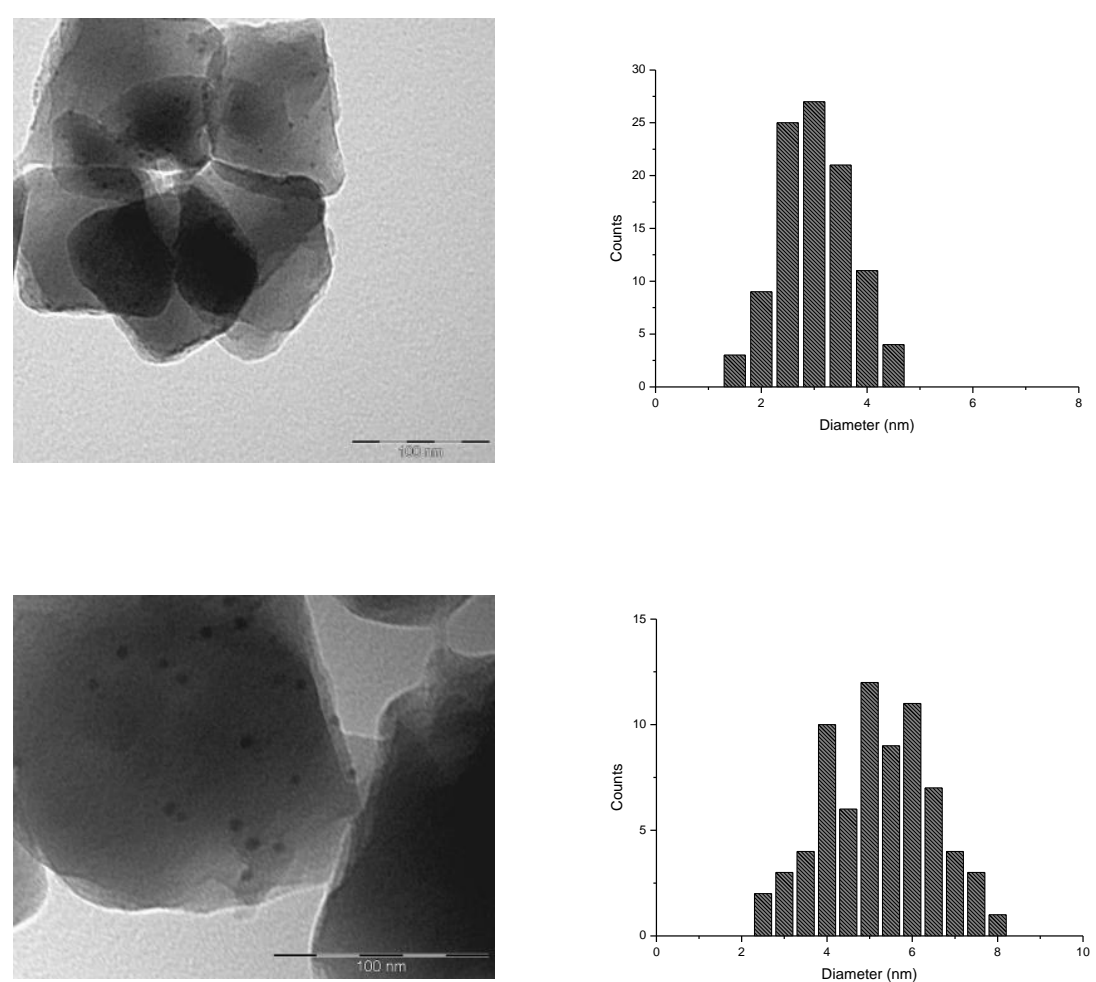

Fig. S2. TEM images and particle size distributions of (top) fresh and (bottom) used Pd@MIL101. 


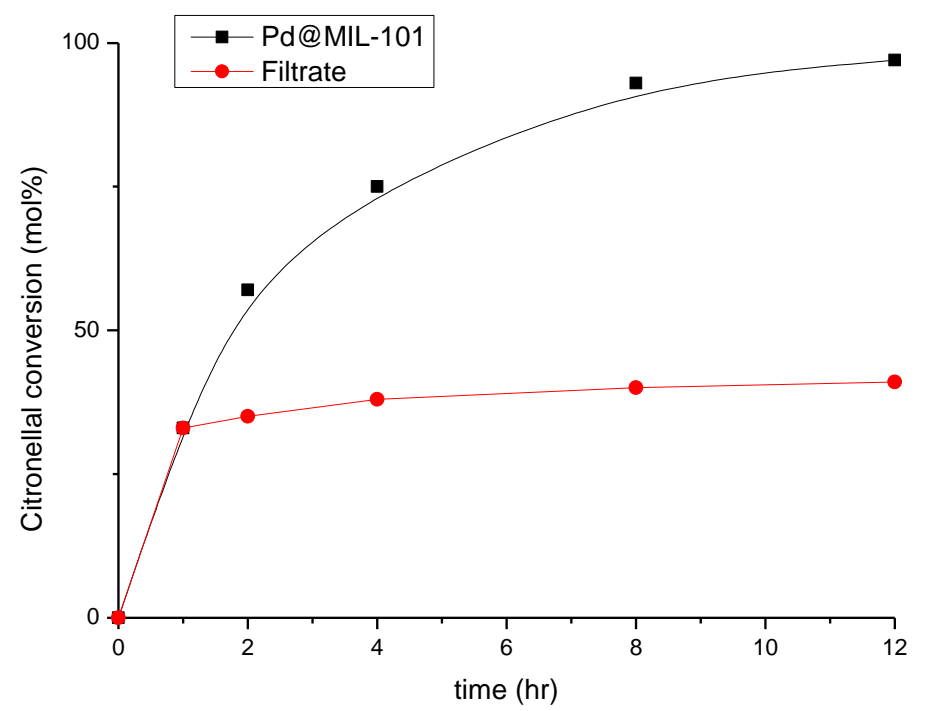

Fig. S3. Hot filtration test. Black curve: Conversion of citronella over Pd@MIL-101. Red curve: The reaction was carried out in the presence of Pd@MIL-101 for 1 hr. Then the catalysts was removed by filtration at the reaction temperature and the reaction was followed in the filtrate at the same temperature.

' G. Ferey, C. Mellot-Draznieks, C. Serre, F. Millange, J. Dutour, S. Surble and I. Margiolaki, Science, 2005, 309, 2040-2042.

ii Y. Pan, B. Yuan, Y. Li and D. He, Chem. Commun., 2010, 46, 2280-2282. 